\title{
EMBEDDING THE DIAMOND LATTICE IN THE RECURSIVELY ENUMERABLE TRUTH-TABLE DEGREES
}

\author{
CARL G. JOCKUSCH, JR. ${ }^{1}$ AND JEANLEAH MOHRHERR
}

\begin{abstract}
It is shown that the four element Boolean algebra can be embedded in the recursively enumerable truth-table degrees with least and greatest elements preserved. Corresponding results for other lattices and other reducibilites are also discussed.
\end{abstract}

For sets $A, B \subseteq \omega$, we say that $A$ is a truth-table (tt) reducible to $B$ if there exists an effective procedure for reducing any question of the form " $n \in A$ ?" to an equivalent finite Boolean combination of questions of the form " $k \in B$ ?" Then, $A, B$ are said to have the same $\mathrm{tt}$-degree if each is tt-reducible to the other, and $\mathrm{tt}$-degrees have a natural ordering induced by tt-reducibility. (See [1, 6 and 8] for information on tt-degrees.) We show the existence of two incomparable recursively enumerable (r.e.) $\mathrm{tt}$-degrees with supremum $\mathbf{0}^{\prime}$ (the highest r.e. $\mathrm{tt}$-degree) and infimum $\mathbf{0}$ (the lowest). In other words, the four-element Boolean algebra (known also as the diamond lattice) can be embedded as a lattice in the r.e. tt-degrees with least and greatest elements preserved. We also obtain analogous results with the diamond lattice replaced by each of the two five-element nondistributive lattices (pentagon and 1-3-1) and with tt-reducibility replaced by many of its restricted forms, such as bounded truth-table and positive reducibility [2].

The history of this problem is as follows. A. H. Lachlan proved in his well-known "nondiamond theorem" [5, Theorem 5] that the diamond lattice cannot be embedded in the r.e. Turing degrees with 0 and 1 preserved. His proof simultaneously establishes the corresponding result for r.e. weak truth-table (wtt) degrees [6]. Lachlan also showed in [4] that no two incomparable r.e. many-one $(m)$ degrees can have supremum $\mathbf{0}^{\prime}$, so the diamond lattice cannot be embedded in the r.e. $m$-degrees with 1 preserved. The trend of these results makes it reasonable to conjecture that the diamond lattice cannot be embedded in the r.e. tt-degrees with 0 and 1 preserved, although in the other direction D. Posner [7] proved that the Turing degrees below $0^{\prime}$ are complemented. In [6, Theorem 6.6] P. G. Odifreddi announced that in fact the diamond lattice can be embedded in the r.e. $\mathrm{tt}$-degrees with 0 and 1 preserved. His construction involved splitting a creative set $K$ into two disjoint r.e.

Received by the editors April 9, 1984.

1980 Mathematics Subject Classification. Primary 03D30; Secondary 03D25.

Key words and phrases. Recursively enumerable sets, truth-table degrees, lattice embeddings.

${ }^{1}$ This research was partially supported by the Science and Engineering Research Council of the U. K. and by the National Science Foundation.

(C)1985 American Mathematical Society $0002-9939 / 85 \$ 1.00+\$ .25$ per page 
sets $A, B$ in such a way that every $C$ tt-reducible to each of $A, B$ is recursive. However, as R. Shore pointed out, there is a serious difficulty with his sketched proof since the strategy for a given set $C$ as above, with given potential tt-reductions from $C$ to $A, B$, may permanently restrain infinitely many numbers from $A$ in the case when $C$ is recursive. This restraint may force $A$ to be recursive, so that the incomparability requirements cannot then be satisfied. Our proof uses the basic approach devised by Odifreddi, but we modify his strategy to ensure that each requirement imposes only a finite amount of restraint over the entire construction. The modification involves giving up the disjointness of $A$ and $B$ and also making strong use of the truth-table nature of the reductions by checking in advance the effect on $C$ (under the given truth-table reductions) of putting a given number into $A$ or $B$ (or both). We still do not know whether there are disjoint tt-incomparable r.e. sets $A, B$, such that $A \cup B$ is creative and every r.e. set $C$ tt-reducible to each of $A, B$ is recursive.

Our results combined with those of Lachlan already mentioned solve the problem of the embeddability of the diamond lattice (preserving 0 and 1) in the r.e. degrees for almost every reducibility between many-one and Turing reducibility that has been studied. What emerges is a curious pattern of negative results for the strongest and weakest reducibilities ( $m$, wtt and $T$ ) and positive results for intermediate ones $(\mathrm{tt}, \mathrm{btt}$, and $p$ ). Thus it is not clear whether embeddability or nonembeddability is more "pathological."

We are grateful to P. Fejer for helpful conversations on the subject of this paper.

Our notation is quite standard. We let $\varphi_{e},\{e\}^{A}$ be the $e$ th partial recursive function and the $e$ th $A$-partial recursive function, respectively. If $\varphi_{i}(x)$ is defined, we let $[i]^{A}(x)=1$ if $A$ satisfies the truth-table condition with index $\varphi_{i}(x)$ (denoted $\left.A \vDash \varphi_{i}(x)\right)$ and $[i]^{A}(x)=0$ if $A \not \varphi_{i}(x)$. Thus the sets truth-table reducible to $A$ are precisely those whose characteristic functions are of the form $[i]^{A}$ for some $i$ with $\varphi_{i}$ total. We henceforth identify sets with their characteristic functions. We write $\{e\}_{s}^{A},[i]_{s}^{A}$ for the portions of $\{e\}^{A},[i]^{A}$ respectively which can be computed in at most $s$ steps.

THEOREM. For any r.e. set $D$, there are r.e. sets $A, B$ such that $A \cup B=D, A^{\prime} \leqslant T$ $0^{\prime}, B^{\prime} \leqslant{ }_{T} 0^{\prime}$, and every set tt-reducible to each of $A, B$ is recursive.

Proof. We enumerate $D$ so that at each stage $s$ exactly one new element, denoted $d_{s}$, appears in $D$. At stage $s, d_{s}$ is enumerated in $A$ or $B$ or both, and no other element enters $A$ or $B$. Hence $D=A \cup B$. At the same time we attempt to satisfy the obvious negative requirements:

$N_{e}(A)$ : Preserve the computation of $\{e\}_{s}^{A^{s}}(e)$ by $A$-restraint, if it is convergent.

$N_{e}(B)$ : Preserve $\{e\}_{s}^{B^{s}}(e)$ by $B$-restraint.

$Q(i, j)$ : If $[i]^{A}=[j]^{B}=f(f$ total $)$, then $f$ is recursive.

As usual, these requirements are assigned a priority ranking, say $R_{3 e}=N_{e}(A)$, $R_{3 e+1}=N_{e}(B)$, and $R_{3<i, j>+2}=Q(i, j)$. As in the Sacks splitting theorem [10, Theorem 2.5], we let $e_{s}$ be the least $e \leqslant s$ such that enumeration of $d_{s}$ in $A$ or $B$ might affect $R_{e}$ and choose the action at stage $s$ so as to preserve $R_{e_{s}}$. It is obvious 
how this is to be done if $R_{e_{s}}$ is $N_{e}(A)$ or $N_{e}(B)$. However there is a difficulty with $Q(i, j)$ because it restrains both $A$ and $B$, and it is not possible to preserve both restraints. In addition, we must choose our strategy for $Q(i, j)$ so that it acts only finitely often over the entire construction. Thus the strategy of preserving agreements between the apparent values of $[i]^{A},[j]^{B}$ (as used in the usual r.e. Turing minimal pair construction [10, Theorem 4.2]) is not suitable. Instead we only preserve (or create) apparent disagreements between $[i]^{A}$ and $[j]^{B}$. We then show that if no disagreements between $[i]^{A}$ and $[j]^{B}$ are permanently preserved and these are total, then they are recursive.

Construction. At stage $s$, we assume $A^{s}, B^{s}$ have been defined and define $A^{s+1}$, $B^{s+1}$. Let $d_{s}$ be the unique number enumerated in $D$ at stage $s$. We say that $N_{e}(A)$ is affected by $d_{s}$ if $\{e\}_{s}^{A^{s}}(e)$ is defined and $d_{s}$ is less than the use of this computation. This is defined analogously for $N_{e}(B)$. We say that $Q(i, j)$ is affected by $d_{s}$ if there is an $x \leqslant s$ such that $[i]_{s}^{A^{s}}(y),[j]_{s}^{B^{s}}(y)$ are defined for all $y \leqslant x,[i]^{A^{s}} \uparrow x=[j]^{B^{s}} \uparrow x$, and further either

$$
A^{s} \cup\left\{d_{s}\right\} \vDash \varphi_{i}(x) \text { iff } A^{s} \not \vDash \varphi_{i}(x)
$$

or

$$
B^{s} \cup\left\{d_{s}\right\} \vDash \varphi_{j}(x) \text { iff } B^{s} \not \vDash \varphi_{j}(x) .
$$

If no $R_{e}, e \leqslant s$, is affected by $d_{s}$, let $A^{s+1}=A^{s} \cup\left\{d_{s}\right\}, B^{s+1}=B^{s}$. Otherwise, let $e_{s}$ be the least $e \leqslant s$ such that $R_{e}$ is affected by $d_{s}$. If $R_{e_{s}}$ is of the form $N_{e}(A)$, let $A^{s+1}=A^{s}, B^{s+1}=B^{s} \cup\left\{d_{s}\right\}$. If $R_{e_{s}}$ is of the form $N_{e}(B)$, let $A^{s+1}=A^{s} \cup\left\{d_{s}\right\}$, $B^{s+1}=B^{s}$.

Finally, suppose that $R_{e_{s}}$ is $Q(i, j)$. Then either (1) or (2) holds. If (1) holds, let $B^{s+1}=B^{s} \cup\left\{d_{s}\right\}$ and choose $A^{s+1}$ to be either $A^{s}$ or $A^{s} \cup\left\{d_{s}\right\}$ in such a way that

$$
A^{s+1} \vDash \varphi_{i}(x) \Leftrightarrow B^{s+1} \not \varphi_{j}(x) .
$$

This is possible because (1) holds. If (1) does not hold, proceed analogously, replacing $A$ by $B$ and $i$ by $j$. Then (3) still can be achieved because (2) holds. This concludes the construction.

It is clear the $A, B$ are r.e. sets and $A \cup B=D$.

LEMMA 1. For each $e$, there are only finitely many $s$ with $e_{s}=e$.

Proof. As usual the proof is by induction on $e$. The induction step is standard if $R_{e}$ is of the form $N_{i}(A)$ or $N_{i}(B)$. Assume now that $R_{e}$ is $Q(i, j)$, and choose $s_{0}$ so that $e_{s}<e$ holds for no $s \geqslant s_{0}$. Assume for a contradiction that $e_{s}=e$ for infinitely many $s$. If $e_{s}=e$, let $x_{s}$ denote the value of $x$ used in the construction. By (3) and the clause $[i]^{A^{s}} \uparrow x=[j]^{B^{s}} \uparrow x$ we see that $x_{s}$ is nonincreasing in $s$ for $s \geqslant s_{0}, e_{s}=e$. Thus there exists $x^{*}$ such that $x_{s}=x^{*}$ for all sufficiently large $s$ with $e_{s}=e$. However, for sufficiently large $s, d_{s}$ is not involved in the truth-table conditions $\varphi_{i}\left(x^{*}\right)$ or $\varphi_{j}\left(x^{*}\right)$, so that $d_{s}$ cannot affect $Q(i, j)$. This contradiction establishes the lemma.

From the lemma and the construction it follows that $e \in A^{\prime}$ whenever $\{s$ : $\{e\}_{s}^{A^{s}}(e)$ defined $\}$ is infinite. Hence $A^{\prime} \leqslant{ }_{T} K$ by the limit lemma, and $B^{\prime} \leqslant{ }_{T} K$ by a similar argument. 
LemMa 2. If $[i]^{A}=[j]^{B}=E$, then $E$ is recursive.

Proof. Let $R_{e}=Q(i, j)$ and choose $s_{0} \geqslant e$ so that $e_{s} \leqslant e$ holds for no $s \geqslant s_{0}$. To compute $E(x)$ simply search for $s \geqslant s_{0}, x$ with $[i]_{s}^{A^{s}}(y),[j]_{s}^{B^{s}}(y)$ defined and equal for all $y \leqslant x$, and then $E(x)=[i]_{s}^{A^{s}}(x)$. To see that this is correct, it suffices to show

$$
[i]_{s}^{A^{s}}(x)=[i]_{t}^{A^{t+1}}(x)=[j]_{t}^{B^{t+1}}(x)
$$

whenever $x, s_{0} \leqslant s \leqslant t$ and $[i]_{s}^{A^{s}}(y)=[j]_{s}^{B^{s}}(y)$ for all $y \leqslant x$. Suppose for a contradiction that (4) fails and let $x_{0}$ be the least $x$ for which it fails. Fix any $s \geqslant s_{0}$ for which (4) fails with $x=x_{0}$, and let $t_{0}$ be the least $t \geqslant s_{0}$ for which it fails. Then, by minimality of $x_{0},[i]_{t}^{A^{\prime}}(y)$ and $[j]_{t}^{B^{\prime}}(y)$ are defined and equal for all $y<x_{0}$. By minimality of $t$, we must have $[i]^{A^{t+1}}\left(x_{0}\right) \neq[i]^{A^{t}}\left(x_{0}\right)$ or $[j]^{B^{t+1}}\left(x_{0}\right) \neq[j]^{B^{t}}\left(x_{0}\right)$. Hence $d_{t}$ affects $Q(i, j)$ at $t$, so $e_{t} \leqslant e$. This contradicts the choice of $s_{0}$, so the proof is complete.

COROLLARY 1. The diamond lattice is embeddable in the r.e.truth-table degrees with 0 and 1 preserved.

Proof. Let $D=K$ in the theorem. We have $K=A \cup B \leqslant_{\mathrm{tt}} A \oplus B$, and $A \oplus B \leqslant_{\mathrm{tt}}$ $K$ is automatic. Then $0<_{\mathrm{tt}} A, B<_{\mathrm{tt}} K$ and $A, B$ are tt-incomparable by the preceding and the lowness of $A$ and $B$.

The proof of this corollary establishes the analogous result for a wide class of reducibilities, namely all those intermediate between bounded disjunctive reducibility $\left(\leqslant_{\mathrm{bd}}\right)$ and truth-table reducibility. (We say $A \leqslant_{\mathrm{bd}} B$ if any question of the form " $m \in A$ ?" can be effectively reduced to disjunction $k_{1} \in B \vee \cdots \vee k_{j} \in B$, with $j$ independent of $m$. Clearly, $A \cup B \leqslant_{\mathrm{bd}} A \oplus B$ for all sets $A, B$.)

COROllary 2. The diamond lattice can be embedded, with 0 and 1 preserved, in the r.e. degrees of all of the following reducibilities: bounded disjunctive (also known as bq-reducibility [3]) disjunctive (also known as q-reducibility [9]) bounded positive, positive [2], and bounded truth-table.

The next result shows that the modular five-element nondistributive lattice known as 1-3-1 can be embedded in the r.e. truth-table degrees.

THEOREM 2. There are three pairwise incomparable r.e. truth-table degrees such that any two of them have sup $\mathbf{0}^{\prime}$ and inf $\mathbf{0 .}$

Proof. We use the method of Theorem 1 (with $D=K$ ) to construct low r.e. sets $A, B, C$ such that $K$ is the union of any two of them and such that no nonrecursive set is truth-table reducible to any two of them. Thus whenever a number is enumerated in $K$, it must be put into at least two of the sets $A, B, C$. We have lowness requirements for each set $A, B, C$ and "minimal pair" requirements for each pair from $A, B, C$ and each pair of Gödel numbers $i, j$. As in Theorem 1 , for instance, the requirement $Q(A, B, i, j)$ is that if $[i]^{A}=[j]^{B}=E$, then $E$ is recursive. This is handled essentially as $Q(i, j)$ is handled in Theorem 1 . In particular if $Q(A, B, i, j)$ plays the role of $R_{e_{s}}$ in Theorem 1 , we set $C^{s+1}=C^{s} \cup\left\{d_{s}\right\}$ and define $A^{s+1}, B^{s+1}$ as in the proof of Theorem 1 . The verification that the construction works is the same as in Theorem 1 . 
Theorem 2 extends in an obvious way from triples to $n$-tuples so that the $1-n-1$ lattice is embeddable in the r.e. $\mathrm{tt}$-degrees with 0 and 1 preserved. We now show that the pentagon lattice is also so embeddable.

THEOREM 3. There are low r.e. sets $A, B, C$ such that $C$ is strictly truth-table below $B, A \oplus C$ is truth-table complete, and the truth-table inf of $A, B$ is 0 .

Proof. We make $A, B, C$ low as in Theorem 1. We put every $n$ in $K$ into at least one of $A$ and $C$. Whenever we put $n$ into $C$, put $2 n$ into $B$. To ensure that $B{ }_{\mathrm{tt}} C$, we use odd numbers as witnesses to ensure that $B \neq[e]^{C}$. Again the construction is finite injury.

Let $P$ be the class of lattices which can be embedded in the r.e. truth-table degrees with least and greatest elements preserved. The results of this paper show that the two-atom Boolean algebra and various other finite lattices are in $P$. It is an open question whether all finite lattices are in $P$. However, the methods of this paper do not seem adequate to show that there are any lattices in $P$ which have pairwise incomparable elements $a, b$ and an element $c<1$ such that $(a \cap b) \cup c=1$. For example, we do not know whether the Boolean algebra with three atoms is in $P$. On the other hand, it seems conceivable that any finite lattice not having three elements $a, b, c$ as above may be shown to be in $P$ by combining the methods of this paper with those of Fejer and Shore [1]. In particular, it is easy to see that the so-called "double-diamond" lattice (obtained by identifying the greatest element of one diamond with the least element of another) is in $P$. This gives an example of a lattice in $P$ having two incomparable elements with a nonzero infimum.

We close with a side remark on bounded disjunctive reducibility, which was defined just before the statement of Corollary 2. It was proved independently by P. R. Young [11, Part I] and A. H. Lachlan [3, Theorem 9] that there exist noncreative sets which are bd-complete. We give a simpler proof here. Let $A$ and $B$ be noncreative r.e. sets with $A \cup B=K$. (The existence of such $A$ and $B$ follows from Theorem 1 and also from the Sacks splitting theorem [10, Theorem 2.5].) Then $A \oplus B$ is bd-complete since $K=A \cup B \leqslant_{\mathrm{bd}} A \oplus B$. On the other hand, $A \oplus B$ cannot be creative since then either $A$ or $B$ would be creative, by Lachlan's universal set theorem [4].

\section{REFERENCES}

1. P. Fejer and R. Shore, Embedding recursively presented lattices into the r.e. tt-degrees (to appear).

2. C. Jockusch, Semirecursive sets and positive reducibility, Trans. Amer. Math. Soc. 131 (1968), $420-436$.

3. A. H. Lachlan, Some notions of reducibility and productiveness, Z. Math. Logik Grundlagen Math. 11 (1965), 17-44.

4. __ A note on universal sets, J. Symbolic Logic 31 (1966), 573-574.

5 . Lower bounds for pairs of recursively enumerable degrees, Proc. London Math. Soc. 16 (1966), 537-569.

6. P. G. Odifreddi, Strong reducibilities, Bull. Amer. Math. Soc. (N.S.) 4 (1981), 37-86.

7. D. Posner, The upper semilattice of degrees $\leqslant 0^{\prime}$ is complemented, J. Symbolic Logic 46 (1981), 705-713.

8. H. Rogers, Theory of recursive functions and effective computability, McGraw-Hill, New York, 1968. 
9. J. R. Shoenfield, Quasicreative sets, Proc. Amer. Math. Soc. 8 (1957), 964-967.

10. R. I. Soare, Fundamental methods for constructing recursively enumerable degrees, Recursion Theory, Its Generalisations and Applications (F. R. Drake and S. S. Wainer, editors), Cambridge Univ. Press, Cambridge, 1980, pp. 1-51.

11. P. R. Young, On semicylinders, splinters, and bounded truth-table reducibility, Trans. Amer. Math. Soc. 115 (1965), 329-339.

Department of Mathematics, University of Illinois, 1409 W. Green Street, Urbana, Illinois 61801

Department of Computer Science, Northern Illinois University, DeKalb, Illinois 60115 